\title{
Ist Regieren „schwieriger“ geworden? - Wahlerfolge und veränderte Rahmenbedingungen für Regierungsparteien bei Bundestagswahlen 1953 bis 2009
}

\author{
Danny Schindler
}

Heutzutage herrsche das Prinzip „Wer regiert, verliert“ meinte Peter Müller recht apodiktisch in einem seiner letzten Interviews als Ministerpräsident des Saarlands. ${ }^{1}$ Allein die Tatsache, dass Parteien Regierungsverantwortung übernehmen, ist aber nicht per se kausal für deren Ergebnisse bei Folgewahlen; wichtiger ist vielmehr, wie sie mit einer übernommenen Regierungsverantwortung umgehen. Liefern Regierungen - im Kontrast zu einer öffentlich kritisierenden und Alternativen aufzeigenden Opposition - in den Augen der Wähler eine schlechte Leistung ab, werden sie bei der nächsten Wahl durchaus abgestraft. Intuitiv mag man Müllers Aussage gerade angesichts einer ganzen Reihe an Krisendiagnosen (Reform-, Steuerungs-, Finanz-, Schuldenkrise etc.) allerdings zustimmen.

Insbesondere rufen wahrgenommene Krisensituationen in der öffentlichen Debatte laute Klagen über mangelnde politische Führung hervor. ${ }^{2}$ Das dem Repräsentationsprinzip innewohnende Spannungsverhältnis von Führung und Gefolgschaft gilt insofern zwar für alle Parteien. Besonders offenbar wird es indessen für jene, die Regierungs- und damit Entscheidungsverantwortung tragen ${ }^{3}-$ und als Ausweis von Führungsleistung in diesem Sinne etwa Interessengegensätze ausgleichen, zukünftige Probleme antizipieren oder Repräsentierte auch bei mehrheitlich abweichenden (Ausgangs-)Präferenzen von ihren Entscheidungen überzeugen müssen.

Ist es heute nun aber, wie es das oben genannte Zitat impliziert, tatsächlich mühsamer, Zustimmung für die eigene Regierungsarbeit zu gewinnen? Ist - plakativ formuliert - Regieren also „schwieriger“ geworden? Will man entsprechende Vermutungen auf empirische Anhaltspunkte stützen, bietet sich ein Blick auf die Wahlergebnisse von Regierungsparteien an. ${ }^{4}$ Aufschlussreich ist vor allem, ob sich hier Veränderungen im historischen Verlauf und

1 Interview mit Peter Müller, in: Cicero, 8. Jg. (2011), H. 8, S. 26 - 29, S. 28.

2 Vgl. etwa den von Jürgen Habermas, Ulrich Beck und anderen unterzeichneten offenen Brief zur Krise der Eurozone: Für eine engere politische Union, in: Zeit online vom 23. Juni 2011, http:// www.zeit.de/wirtschaft/2011-06/offener-brief-griechenland-europa (Abruf am 23. September 2011).

3 Für andere verbindlich zu entscheiden ist insofern Kernbestand und Wesensmerkmal politischer Repräsentation. Vgl. Suzanne S. Schüttemeyer, Fraktionen im Deutschen Bundestag 1949 - 1997. Empirische Befunde und theoretische Folgerungen, Wiesbaden 1998, S. 22 f.; Ernst Fraenkel, Deutschland und die westlichen Demokratien, Frankfurt am Main 1979, S. 113.

4 Dass zu den vielfältigen Größen, die direkt oder indirekt auf die Wahlentscheidung einwirken, die Regierungspolitik der vergangenen Wahlperiode gehört, dürfte eine feststehende Erkenntnis der Wahlforschung sein. Unabhängig vom jeweiligen Modell des Wahlverhaltens gilt für Demokratien allgemein: Wahlergebnisse hängen davon ab, inwiefern es Regierungen gelingt, politische Probleme durch konsens- oder mehrheitsfähige Entscheidungen zu lösen, sowie vom Erfolg der Opposition, die Wähler durch Kritik, Kontrolle und alternative Problemlösungen zu überzeugen. Vgl. Bernard Manin / Adam Przeworski / Susan C. Stokes, Elections and Representation, in: dies. (Hrsg.), Democracy, Accountability, and Representation, Cambridge 1999, S. 29 - 54; Wilhelm Bürklin / Markus Klein, Wahlen und Wählerverhalten, Opladen 1998, S. 12 f. 
unabhängig von parteispezifischen Mustern ergeben. Die großen Stimmengewinne für die CDU Konrad Adenauers bei der zweiten und die großen Stimmenverluste für die SPD bei der letzten Bundestagswahl mögen außergewöhnlich erscheinen; es stellt sich aber die Frage, ob es auch eine Trendentwicklung zwischen diesen Wegmarken gibt. Bekannt ist in der Wahlforschung zudem, dass etwa bei der so genannten Nebenwahl zum Europäischen Parlament „a pattern of anti-government swing "5 üblich ist, und dass Verluste für nationale Regierungsparteien tendenziell ansteigen. Gilt dies ähnlich auch für nationale Parlamentswahlen in Deutschland, also eine Hauptwahl?

Gegenstand sind im Folgenden die Zweitstimmenergebnisse der Regierungsparteien bei Bundestagswahlen und die Veränderungen im Vergleich zur jeweils vorherigen Wahl. Da die erste Bundestagswahl nur für Berechnungszwecke einbezogen wird, verbleiben für die Untersuchung die 16 (Folge-)Wahlen seit 1953.6 Als Datengrundlage für alle Berechnungen und Darstellungen dienten die amtliche Wahlstatistik sowie das Datenhandbuch des Deutschen Bundestages.

\section{Wahlergebnisse von Regierungsparteien bei Bundestagswahlen}

Bevor entsprechende Ergebnisse ausgewiesen werden, ist auf zwei Aspekte hinzuweisen. Zum einen ist die Datenbasis mit 16 Wahlen schmal. Zum anderen haben sich im Untersuchungszeitraum auch Veränderungen im Parteiensystem vollzogen. Von Belang ist in dieser Hinsicht aus der Perspektive der Wähler insbesondere die Zahl der im Bundestag vertretenen beziehungsweise für eine parlamentarische Vertretung relevanten Parteien. Dabei zeichnet sich diese „Angebotsseite“ nach Abschluss der Neuformierungsphase in den 1950 er Jahren zwar über weite Strecken durch eine große Stabilität aus. Wesentliche Einschnitte stellten aber die parlamentarische Etablierung der Grünen 1983 sowie der PDS (heute Linkspartei) seit 1990 dar. $^{7}$

\subsection{Wahlergebnisse für Regierungskoalition, Kanzlerpartei und kleine Regierungspartei}

Die Veränderungen der Wahlergebnisse für die jeweilige Regierungskoalition insgesamt zeigen ein deutliches Bild (vgl. Abbildung 1). Gab es in den ersten Jahrzehnten der Bundesrepublik noch vergleichsweise häufig einen kumulierten Stimmengewinn für die Regierungsparteien, ist dies seither die Ausnahme. Bis zur Mitte des Untersuchungszeitraums 1980 sind zum Beispiel in fünf von acht Folgewahlen Gewinne zu verzeichnen, in den acht Wahlen seit 1983 war dies nur einmal (1990) der Fall. Seit 1994 kann kein kumulierter Gewinn

5 Michael Marsh, Testing the Second-Order Election Model after Four European Elections, in: British Journal of Political Science, 28. Jg. (1998), H. 4, S. 591 - 607, S. 606.

6 Eine Ausdehnung auf Landtagswahlen wurde wegen der im Einzelfall unterschiedlichen und damit im Gesamtbild schwer zu interpretierenden Einflusseffekte der Bundes- auf die Landesebene bewusst unterlassen. Vgl. hierzu zum Beispiel das Schwerpunktheft der ZParl, 38. Jg. (2007), H. 3.

7 Wenngleich Grüne und PDS 1990, die PDS auch 1994 und 2002 nicht als Fraktion in den Bundestag einziehen konnten (1990 beziehungsweise 1994 hatten sie jedoch Gruppenstatus), blieben sie bei den jeweiligen Wahlen auf der Ebene der Wählerwahrnehmung doch als Partei mit echter Chance auf Vertretung im Parlament relevant. 
mehr festgestellt werden. Dies ist auch die längste Phase ohne Gewinne für eine Regierungskoalition: Während es in den 1960er Jahren $(1961,1965)$ und in den 1980er Jahren $(1983,1987)$ jeweils zwei aufeinanderfolgende Wahlen mit Verlusten gab, sind es seit 1994 nunmehr fünf.

Ein ähnliches Bild offenbart das Ausmaß der Stimmengewinne und -verluste. Nicht nur finden sich Maximalwerte an den Enden des Untersuchungszeitraums: die größten Gewinne wurden 1953, die größten Verluste 2009 erzielt. Auch sind höhere Einbußen vor allem seit 1983 feststellbar: Für die drei „Verlust“-Wahlen vor 1983 ergibt sich einmal ein Minus von 4,8 Prozentpunkten (1961), das gleichzeitig die maximalen Verluste in diesem Zeitraum anzeigt; die sieben „Verlust“-Wahlen seit 1983 zeigen fünfmal ein Minus von mindestens 4,8 Prozentpunkten, in vier Fällen davon sind die Einbußen weitaus größer (1983, 1994, 1998, 2009). Betrachtet man lediglich die Gewinne, ist - passend dazu - der nur einmal auftretende Zugewinn einer Regierungskoalition seit 1983 (1990) auch der geringste im Untersuchungszeitraum überhaupt. Insgesamt zeigt Abbildung 1 so gesehen eine zyklische Abwärtsentwicklung.

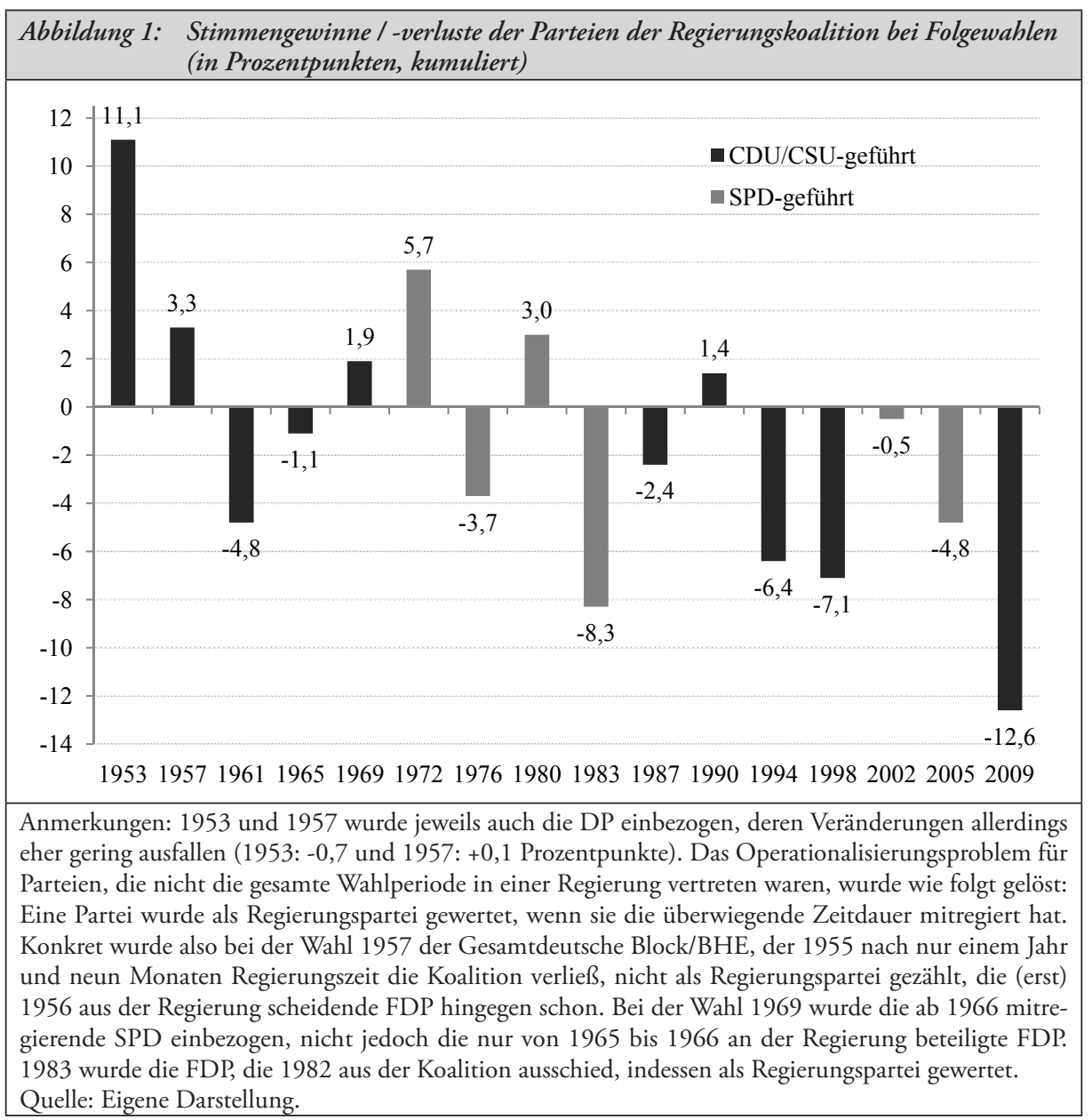


Parteispezifische Besonderheiten sind im Hinblick auf die Frage, welche Partei den Kanzler stellt, im Übrigen nicht auszumachen. CDU- und SPD-geführte Bundesregierungen reihen sich gleichermaßen in die beschriebene Entwicklung ein. Ähnliches gilt auch für das Koalitionsformat einer Großen Koalition. Dem kumulierten Gewinn 1969 steht ein Verlust 2009 gegenüber.

Die weitere Differenzierung zeigt bei der jeweiligen Kanzlerpartei zunächst ebenfalls eine Verschiebung hin zu fortwährenden Verlusten (vgl. Abbildung 2). Der letzte Zugewinn einer großen Partei aus der Regierungsverantwortung heraus liegt bereits über 30 Jahre zurück (1980). Die Union als häufigste Kanzlerpartei konnte sich gar 1965 das letzte Mal bei einer Folgewahl verbessern. Auch hier deutet sich also - wie schon bei der Betrachtung der Koalition insgesamt - eine gewisse Zweiteilung des Untersuchungszeitraums an: Während in den acht Wahlen bis einschließlich 1980 in der Mehrzahl (fünfmal) Zugewinne zu verzeichnen sind, haben die Kanzlerparteien in den acht Wahlen danach stets verloren. Ebenso wie bei der Betrachtung der Regierungskoalition gilt diese Entwicklung wiederum unabhängig von der Parteifarbe. Beide großen Parteien haben in der Mehrzahl der Fälle verloren (die Union in sieben von zehn, die SPD in vier von sechs Folgewahlen), beide konnten seit der Wahl 1980 in Regierungsverantwortung keine Stimmengewinne mehr erzielen. Die größten Verluste fallen auf die letzte Amtsperiode der Regierung Kohl (1998). Aus einer Großen Koalition heraus (1969, 2009) haben Kanzlerparteien immer Einbußen hinnehmen müssen.

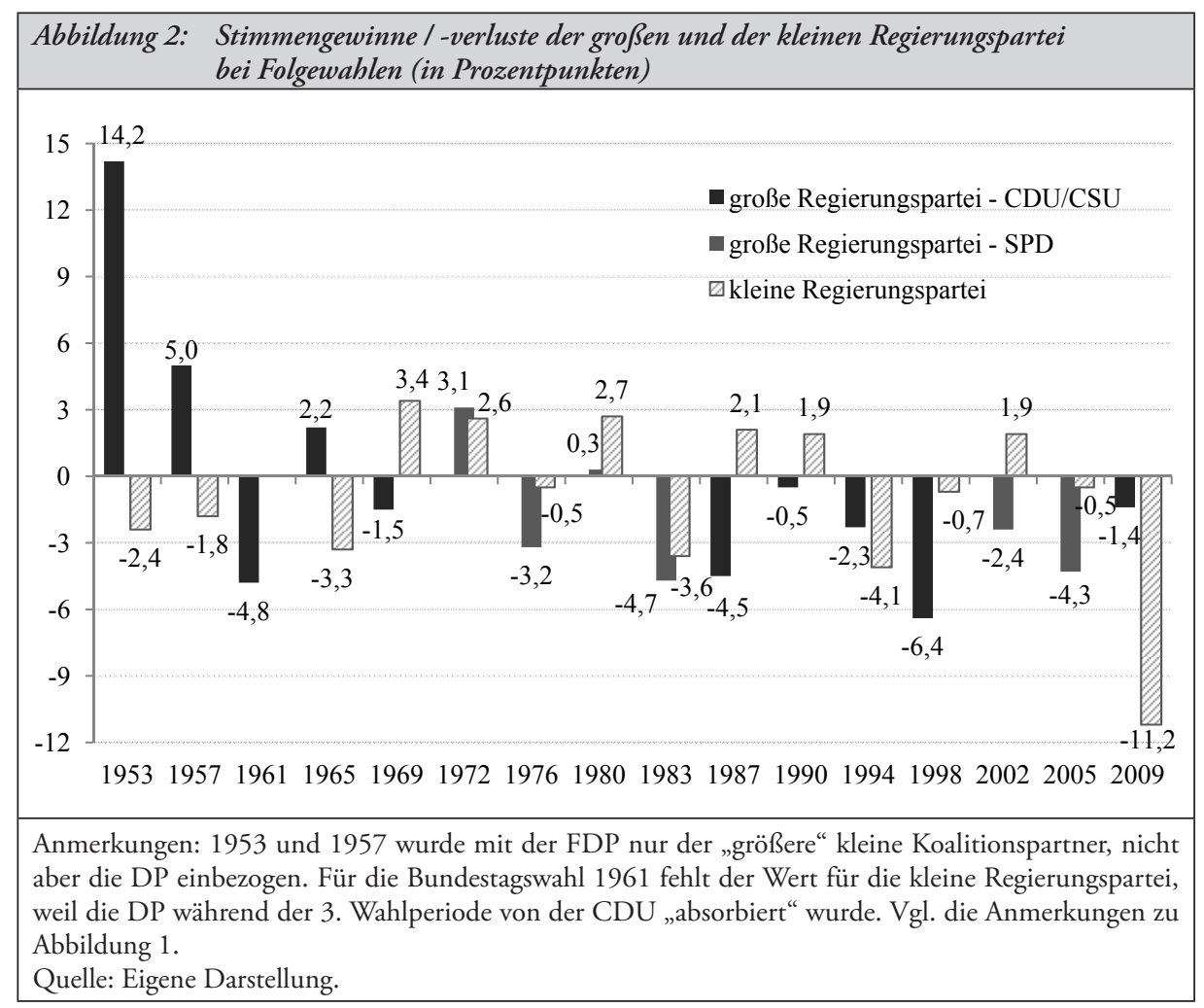


Kein eindeutiges Bild ergibt sich hingegen für die kleinen Regierungsparteien (vgl. Abbildung 2). Zwar verloren auch diese in der Mehrzahl aus der Regierungsverantwortung heraus - neun Fälle von Verlusten stehen hier sechsmal Gewinne gegenüber. Allerdings ist eine mit den Kanzlerparteien vergleichbare Abwärtsentwicklung nicht feststellbar. Deutlich wird zunächst, dass die kleine Regierungspartei, teilweise im Gegensatz zu ihrem großen Koalitionspartner, in den ersten Jahrzehnten der Bundesrepublik kaum Stimmengewinne zu verzeichnen hatte. Später, vor allem auch noch nach 1980, konnte sie dagegen durchaus zulegen, letztmalig 2002. Dabei finden sich die drei höchsten Gewinne in der ersten Hälfte des Untersuchungszeitraums (bis 1980), die drei geringsten sowie die drei höchsten Verluste in der zweiten. Im Vergleich mit der großen Regierungspartei ist das Minus des kleinen Koalitionspartners - sieht man einmal von der Ausnahme der SPD in der Großen Koalition $2009 \mathrm{ab}$ - insgesamt aber deutlich geringer. Nach den Rekordverlusten der SPD von 2009 liegen die zweithöchsten Einbußen eines Juniorpartners bei 4,1 Prozentpunkten (1994). Die Kanzlerpartei verlor hingegen fünfmal, das heißt in nahezu jeder zweiten Wahl mit Verlusten, mehr als 4,1 Prozentpunkte. Ein Blick nur auf das Format der Großen Koalition (1969, 2009) zeigt, dass hier die jeweiligen Maximalwerte für Veränderungen beim kleinen Regierungspartner liegen: Als Junior in einer Großen Koalition verzeichnete die SPD einmal den höchsten Gewinn unter den kleineren Regierungsparteien, einmal verlor sie mit den höchsten Stimmeneinbußen überhaupt.

Auch hinsichtlich der Anzahl der Regierungsparteien mit Stimmenverlusten wird eine gewisse Zweiteilung sichtbar (vgl. Abbildung 3). So gab es vor 1983 zweimal doppelte Gewinne, also weder Verluste für die große noch die kleine Regierungspartei (1972, 1980), und zwar beide Male unter einer SPD-geführten Regierung. Zudem liegen in diesem Zeitraum fünfmal einseitige Einbußen (von 1953 bis 1969), die dreimal die kleine und zweimal die große Partei betrafen. Einmal verloren auch beide Koalitionspartner (1976), seit 1983 hingegen immer mindestens eine Partei (genauer: mindestens die Kanzlerpartei), wobei insgesamt sogar doppelte Verluste dominieren (1983, 1994, 1998, 2005, 2009). Einbußen nur für den Juniorpartner einer Regierungskoalition traten das letzte Mal 1965 auf. Musste nach der Großen Koalition 1969 lediglich die Union ein Minus hinnehmen, traf es 2009 beide Partner.

\begin{tabular}{|c|c|c|c|c|c|c|c|c|c|c|c|c|c|c|c|}
\hline \multicolumn{16}{|c|}{ Abbildung 3: Regierungsparteien mit Stimmenverlusten bei Folgewablen } \\
\hline $\begin{array}{l}\text { große } \\
\text { Regierungs- } \\
\text { partei }\end{array}$ & & $\bullet$ & & $\bullet$ & & $\bullet$ & & $\bullet$ & $\bullet$ & $\bullet$ & $\bullet$ & $\bullet$ & $\bullet$ & $\bullet$ & $\bullet$ \\
\hline \multirow[t]{2}{*}{$\begin{array}{l}\text { kleine } \\
\text { Regierungs- } \\
\text { partei }\end{array}$} & $\bullet \bullet$ & & & & & $\bullet$ & & $\bullet$ & & & & $\bullet$ & & & • \\
\hline & $\stackrel{\approx}{\cong} \cong$ & 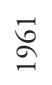 & $\stackrel{\approx}{\varrho}$ & ڤે & & $\stackrel{\circ}{\stackrel{2}{\approx}}$ & $\stackrel{\infty}{\stackrel{\infty}{二}}$ & $\stackrel{\infty}{\curvearrowright}$ & $\stackrel{\infty}{\curvearrowright}$ & 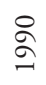 & 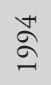 & $\stackrel{\infty}{\check{\sigma}}$ & $\begin{array}{l}\text { ¿ิ } \\
\text { ¿े }\end{array}$ & ڤి & ஓి \\
\hline \multicolumn{16}{|c|}{$\begin{array}{l}\text { Anmerkungen: Grau hinterlegte Felder weisen aus, dass beide Regierungsparteien Stimmenverluste zu } \\
\text { verzeichnen hatten. } 1961 \text { waren Einbußen nur bei der allein regierenden Kanzlerpartei möglich. Vgl. die } \\
\text { Anmerkungen zu Abbildung } 1 \text { und } 2 . \\
\text { Quelle: Eigene Darstellung. }\end{array}$} \\
\hline
\end{tabular}


Als Zwischenfazit kann festgehalten werden, dass es seit 1953 immer häufiger zu Verlusten für die Regierungsparteien bei Folgewahlen kam, wobei dies in der Einzelbetrachtung der Koalitionspartner vor allem für die Kanzlerparteien zutrifft. Für den kleinen Regierungspartner weist in der Längsschnittperspektive hingegen lediglich das Ausmaß der Gewinne und Verluste einen Abwärtstrend auf.

\subsection{Die Großparteien in Regierung und Opposition im Vergleich}

Vor allem die regierenden Großparteien sind also von Stimmenverlusten betroffen. Offen bleibt allerdings, inwiefern eine entsprechende Entwicklung möglicherweise generell für Union und SPD festzustellen ist, das heißt unabhängig von deren Regierungs- oder Oppositionsstatus. Wäre dies der Fall, hätte also die oppositionelle Volkspartei ${ }^{8}$ im Zeitverlauf ebenfalls zunehmend Stimmeneinbußen zu verzeichnen, relativierte dies auch die bisherigen Befunde. Zu fragen ist daher, ob die große Oppositionspartei von den Verlusten der Kanzlerpartei (noch) profitieren kann, oder ob sich hierin ein allgemeiner Niedergang der Großparteien bei Wahlen widerspiegelt. Bekannt ist in dieser Hinsicht auch, dass etwa der kumulierte Anteil der Volksparteien bei Wahlen seit den 1970er Jahren fast durchgängig abgenommen hat. ${ }^{9}$

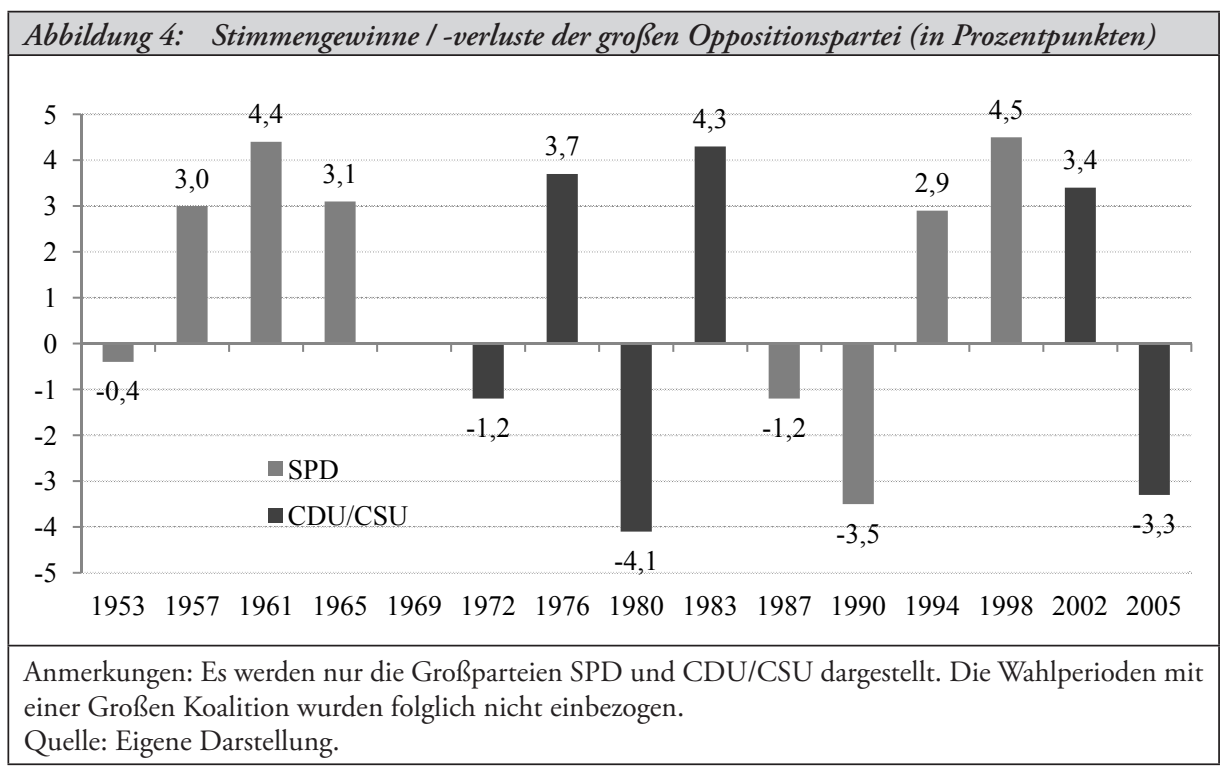

8 Die Begriffe Volkspartei und Großpartei werden hier synonym verwendet, ohne auf die breite politikwissenschaftliche Diskussion um die „Merkmale“ einer Volkspartei einzugehen. Vgl. hierzu etwa Everhard Holtmann, Repräsentation des Volkes durch Volksparteien? Nach wie vor hohes Maß an Parteiidentifikation, in: Eckhard Jesse / Roland Sturm (Hrsg.), Bilanz der Bundestagswahl 2005: Voraussetzungen, Ergebnisse, Folgen, Wiesbaden 2005, S. 211 - 233, S. 212.

9 Vgl. zum Beispiel Richard Hilmer, Bundestagswahl 2009: Ein Wechsel auf Raten, in: ZParl, 41. Jg. (2010), H. 1, S. 147 - 180, S. 149, Abbildung 1; Peter Lösche, Ende der Volksparteien, in: APuZ, B $51 / 2009$, S. $6-12$. 
Die Abbildungen zeigen insgesamt ein komplexes Bild. Aus Abbildung 4 wird zunächst deutlich, dass auch die große Oppositionspartei einige Male Einbußen hinnehmen musste. Dies trifft zwar auf die Wahlen in den ersten zwei Jahrzehnten der Bundesrepublik kaum zu; dennoch lässt sich ein generelles Zeitmuster nicht diagnostizieren. Die oppositionelle Volkspartei konnte auch in den letzten Jahrzehnten (1994 bis 2002) ähnlich große Gewinne erzielen wie bei früheren Wahlen. Parteipolitische Muster lassen sich in dieser Hinsicht nicht ausmachen. Zudem sind Union und SPD in annähernd gleichem Ausmaß von Verlusten und Gewinnen betroffen.

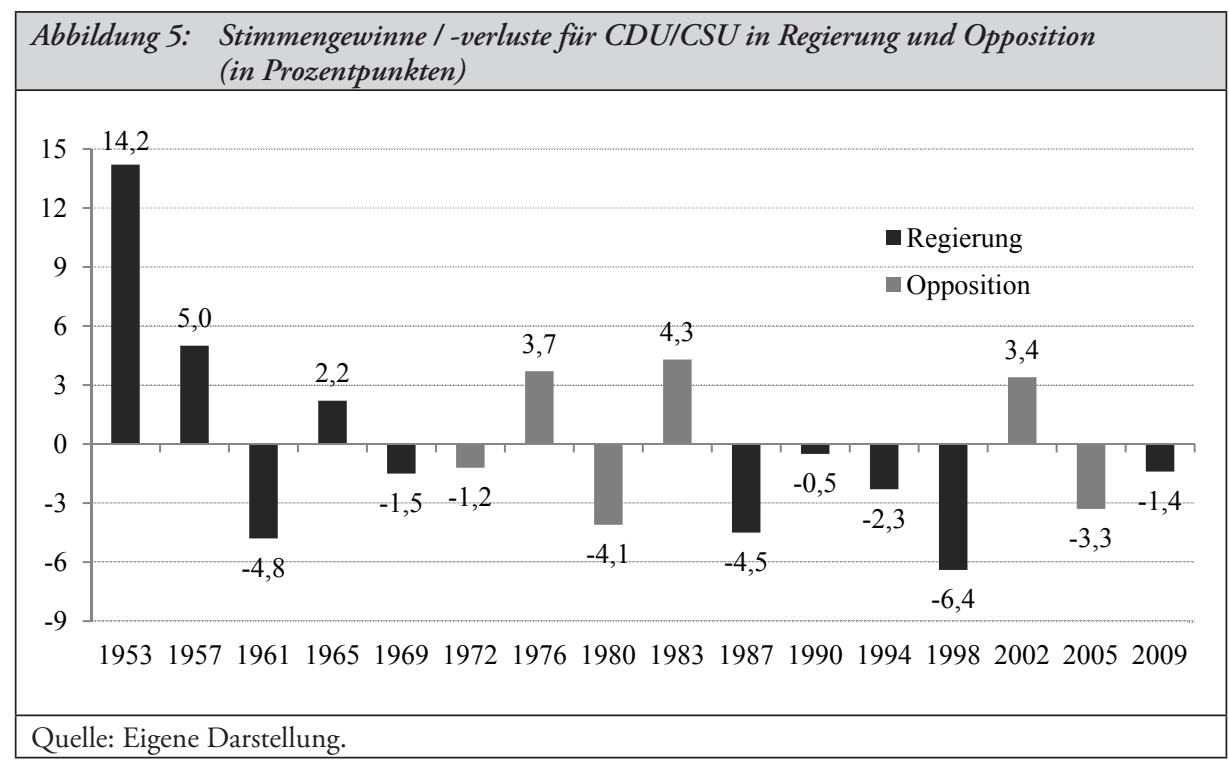

Auch Einzelbetrachtungen beider Großparteien stützen diese Annahmen. So gilt für die Union (vgl. Abbildung 5), dass sie in Regierungsverantwortung nur in den ersten Jahrzehnten der Bundesrepublik Gewinne erzielen konnte. Danach konnte sie zwar noch hinzugewinnen, allerdings nur aus der Opposition heraus.

Auch die SPD konnte nur in den früheren Regierungsbeteiligungen Gewinne verzeichnen (vgl. Abbildung 6), und zwar, wiederum gemäß der oben ausgemachten Zweiteilung des Untersuchungszeitraums, bis 1980 in drei von vier Wahlen, während sie danach in allen vier Wahlen mit Regierungsverantwortung verlor. Allerdings konnte sie auch in der zweiten Hälfte des Untersuchungszeitraums noch hinzugewinnen, dann aber nur in Phasen mit Oppositionsstatus (1994, 1998). Für beide Parteien gilt demnach: Während Gewinn- und Verlustphasen in den Anfangsjahren noch quer zum Regierungs- und Oppositionsstatus lagen, beschränken sich die Gewinne in den letzten Jahrzehnten ausschließlich auf Oppositionszeiten.

Ein erstes Ergebnis lautet also, dass sich zumindest mit den bisherigen Befunden eine generelle Abwärtsbewegung bei Großparteien in der Opposition nicht bestätigen lässt. Im Oppositionsstatus existiert kein Zeitmuster; hier konnten in den letzten Jahrzehnten auch weiter Gewinne erzielt werden. 


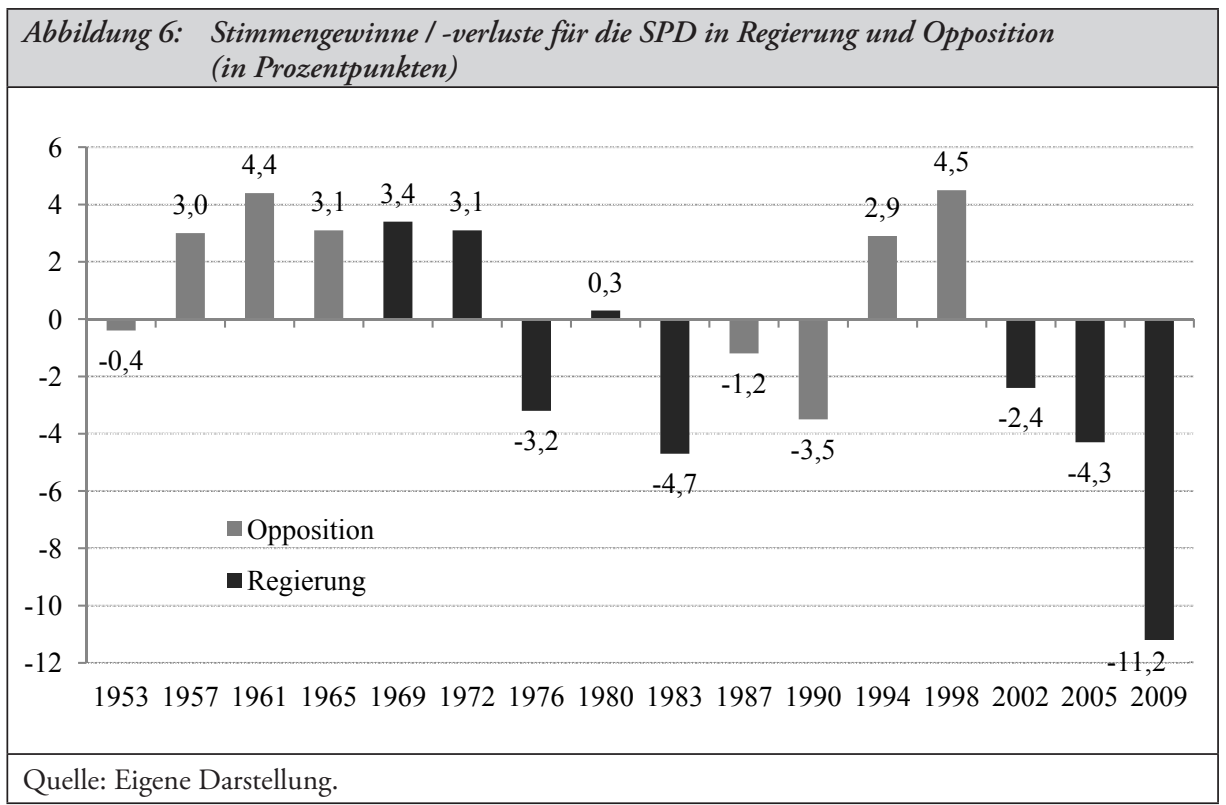

Dem steht im Übrigen auch der in der Wahlforschung konstatierte Rückgang der kumulierten Anteile der Volksparteien seit $1976^{10}$ nicht entgegen. Denn für die Konstellation, in der die große Regierungspartei verliert, die große Oppositionspartei hingegen gewinnt, galt etwa 1983 und 1998, dass die Regierungsverluste die Oppositionsgewinne überstiegen (vgl. Abbildung 7) - und damit im Ergebnis auch zu einem leichten Rückgang der kumulierten Anteile führten. Ein in dieser Hinsicht positiver Saldo ergab sich nur für die Wahlen 1994 und 2002 (die beiden Anstiege der kumulierten Prozentanteile der Großparteien seit 1976). ${ }^{11}$

Allerdings zeigt sich in anderer Hinsicht eine besondere Betroffenheit der Volksparteien. Kontrastiert man nämlich beide Großparteien im Regierungs- und Oppositionsstatus im Zeitverlauf, wird deutlich, dass im Falle von Verlusten für die Kanzlerpartei kein eindeutiges Wechselverhältnis mehr existiert (vgl. Abbildung 7). Ersichtlich ist zunächst, dass bei Wahlen in den ersten zwei Jahrzehnten Union und SPD durchaus zugleich gewinnen konnten $(1957,1965)$, später indes nicht mehr. Überdies gewann bei drei Wahlen bis 1980 sogar nur die große Regierungspartei, während die große Oppositionspartei verlor (1953, 1972, 1980). Auffällig ist nun aber, dass erst seit 1987 beide Großparteien gemeinsam verlieren. Seitdem sind für drei von sechs Wahlen doppelte Stimmenverluste zu verzeichnen, das heißt für die große Regierungs- und die große Oppositionspartei (1987, 1990, 2005). Vorher konnte die opponierende Volkspartei hingegen jedes Mal hinzugewinnen, wenn die

10 Vgl. Richard Hilmer, a.a.O. (Fn. 9), S. 149.

11 Für den Saldo dieser Konstellation - Verluste der großen Regierungs-, Gewinne der großen Oppositionspartei (1961, 1976, 1983, 1994, 1998, 2002) - besteht im Übrigen kein zeitliches Muster: Negative und positive Werte wechseln sich ab (vgl. Abbildung 7). 


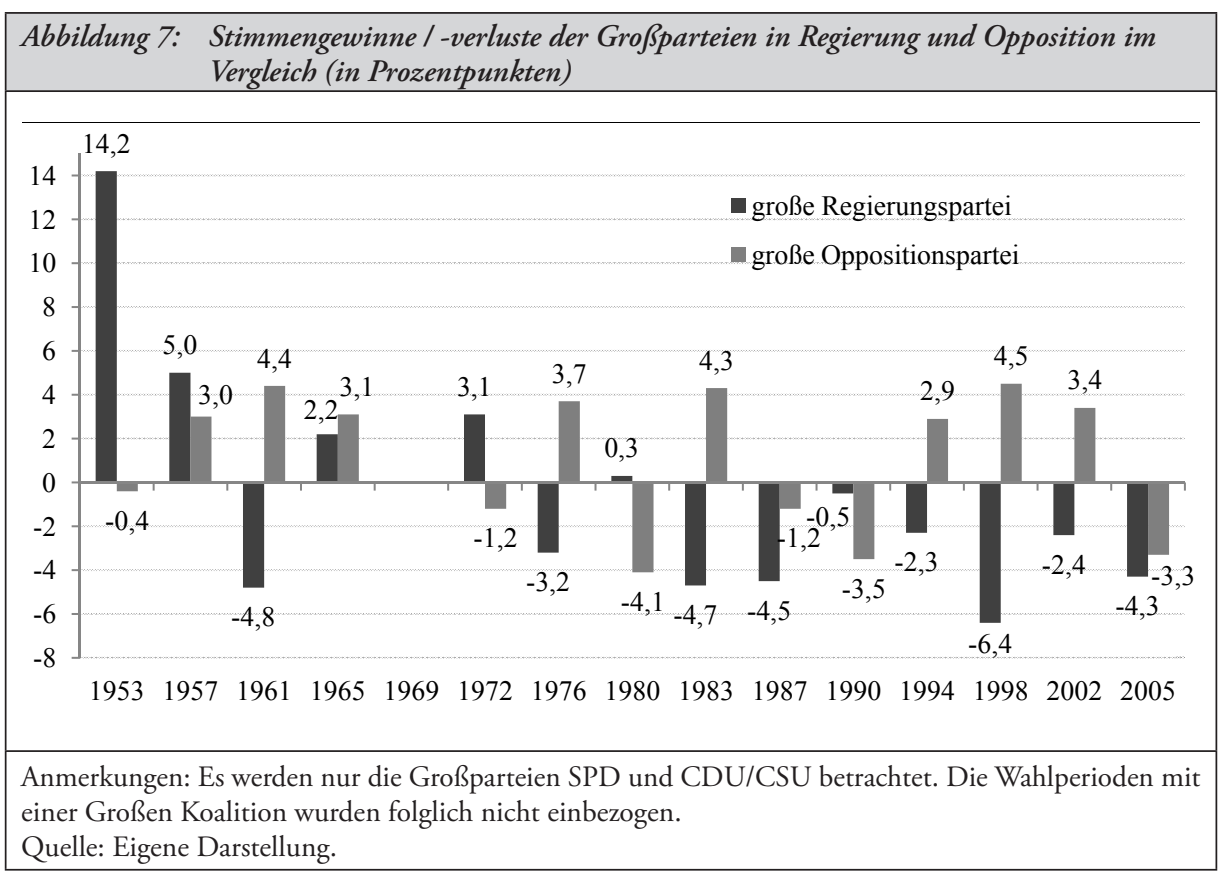

regierende Volkspartei verlor. Aus den Abbildungen 5 und 6 geht somit zwar hervor, dass sich Gewinne bei den Großparteien in den letzten Jahrzehnten auf Oppositionszeiten beschränken. Abbildung 7 zeigt jedoch, dass ein Regierungsmalus mit Blick auf die Großparteien eben nicht mehr automatisch ein Oppositionsbonus ist. ${ }^{12}$ Dieses zweite Ergebnis fügt sich auch in die These eines allgemeinen Niedergangs der Großparteien ${ }^{13}$ ein.

Interessante Ergebnisse ergeben sich im Übrigen auch für die (im Untersuchungszeitraum allerdings nur sechsmal auftretende) Konstellation, dass auf eine Verlustwahl für eine Großpartei vier Jahre später eine Wahl mit Stimmengewinnen folgte. ${ }^{14}$ Dabei waren in vier von sechs Wahlen die Folgegewinne kleiner als die vergangenen Verluste (1965 und 2002 bei der Union, 1980 und 1994 bei der SPD) und damit im Saldo eine Abwärtsbewegung festzustellen. Zweimal war dies nicht der Fall (1976 und 1983 für die Union). Bezieht man den Regierungsstatus ein, wird deutlich, dass bei einer Verlustwahl als Regierungspartei die nachfolgenden Gewinne im Ausmaß immer kleiner waren (1965 und 2002 bei der Union, 1980 bei der SPD). Zugewinne, die die vorherigen Verluste übertrafen, waren demgegenüber nur möglich, wenn man zuvor als Oppositionspartei verloren hatte (1976 und 1983 bei der Union). Nur dann kam es also zu einer „kompensierenden“ Erholung bei Folgewahlen. Blickt man hingegen auf den zeitlichen Verlauf, sind es die letzten drei einschlägigen

12 Auch bei der Kompetenzzuschreibung an Union und SPD wurde dies schon festgestellt. Vgl. Everhard Holtmann, a.a.O. (Fn. 8), S. 221.

13 Vgl. Peter Lösche, a.a.O. (Fn. 9).

14 Eine solche Konstellation trat bei der SPD bei den Wahlen 1980 und 1994 auf, bei der Union 1965, 1976, 1983 und 2002 (jeweils Angabe der Folgewahl mit Stimmengewinnen); vgl. hierzu die Abbildungen 5 und 6. 
Wahlen (1983: Union, 1994: SPD, 2002: Union), für die keine die Verluste kompensierende Gewinnhöhe festgestellt werden kann, auch nicht als oppositionelle Volkspartei (1994 bei der SPD). Allgemein weisen diese Ergebnisse für die Großparteien auf eine zyklische Bewegung mit generell sinkender Tendenz hin, von der die regierenden Volksparteien aber besonders betroffen sind.

\subsection{Fazit: Die Kanzlerparteien als (doppelte) Verlierer}

Insgesamt zeigen die empirischen Befunde der Längsschnittperspektive eine deutliche Verschiebung hin zu Verlusten für die Regierungskoalition insgesamt sowie für die Kanzlerpartei im Besonderen. So konnte die Koalition in der zweiten Hälfte des Untersuchungszeitraums (seit 1983) nur einmal (1990) einen kumulierten Gewinn einfahren, während in der Mehrheit der Fälle seither Verluste von mindestens 4,8 Prozentpunkten zu verzeichnen sind. Mit den fünf Folgewahlen seit 1994 befinden sich Regierungskoalitionen mittlerweile auch in der bislang längsten Phase ohne kumulierten Gewinn. In erster Linie trifft diese Entwicklung dabei die Kanzlerpartei, die vor 1983 in der Mehrzahl der Fälle hinzugewonnen hatte, seither aber bei jeder Wahl verlor.

Demgegenüber ist ein eindeutiger Trend für den kleinen Regierungspartner nicht so einfach nachzuweisen. Zuwächse aus der Regierungsverantwortung heraus wurden hier zum Beispiel auch nach 1980 noch realisiert. Lediglich die Höhe der Gewinne und Verluste zeigt eine leichte Abwärtsbewegung an.

Blickt man auf beide Regierungspartner zugleich, gab es ein Plus sowohl bei der großen als auch der kleinen Regierungspartei nur vor der Bundestagswahl 1983. Seither verlor die große zwar immer, allerdings dominieren für diesen Zeitraum insgesamt auch doppelte Verluste, das heißt Einbußen für beide Regierungsparteien. Alle genannten Entwicklungen vollzogen sich zudem unabhängig von der Parteifarbe: Parteispezifische Besonderheiten sind nicht festzustellen.

Vergleicht man darüber hinaus die Wahlergebnisse für die große Regierungs- und die große Oppositionspartei, ist ein generelles Muster außerhalb der Regierungsverantwortung nicht zu erkennen. Die Volkspartei in der Opposition konnte im Vergleich zur Kanzlerpartei auch in den letzten Jahrzehnten noch Gewinne erzielen und somit von den Verlusten der Regierungspartei profitieren. Dies fügt sich also nicht direkt in die These einer allgemeinen (vom Regierungsstatus unabhängigen) Abwärtsbewegung bei Volksparteien ein und deutet auf eine besondere Betroffenheit der regierenden Großparteien hin. Allerdings ergibt sich auch hier ein zweigeteiltes Bild: Während vor 1987 die opponierende Volkspartei immer hinzugewinnen konnte, wenn die regierende Volkspartei verlor, sind seitdem für drei von sechs Wahlen auch doppelte Verluste, für die große Regierungs- und die große Oppositionspartei zugleich, aufgetreten - ein Befund, der die Annahme eines generellen Abwärtstrends bei den Großparteien stützt. Möglicherweise handelt es sich also um sich wechselseitig verstärkende Prozesse. Das komplexe Bild spricht einerseits dafür, dass es gerade für die regierenden Großparteien schwieriger geworden ist, bei Wahlen $\mathrm{Zu}$ stimmung zu finden. Andererseits ist diese Entwicklung eingebettet in ein besonders schwieriges Umfeld für die Volksparteien allgemein, unabhängig von deren Regierungsoder Oppositionsstatus. Die Kanzlerparteien wären in diesem Zusammenhang also „doppelte" Verlierer. 


\begin{tabular}{|c|c|c|c|c|c|c|}
\hline \multicolumn{7}{|c|}{$\begin{array}{l}\text { Tabelle 1: Durchschnittliche Stimmengewinne /-verluste bei Bundestagswablen } 1953 \text { bis } 2009 \\
\text { (in Prozentpunkten) }\end{array}$} \\
\hline Zeitraum & $\begin{array}{c}\text { Regierungs- } \\
\text { koalition } \\
\text { (kumuliert) }\end{array}$ & $\begin{array}{l}\text { große } \\
\text { Regierungs- } \\
\text { partei }\end{array}$ & $\begin{array}{l}\text { kleine } \\
\text { Regierungs- } \\
\text { partei } \\
\text { (mit SPD) }\end{array}$ & $\begin{array}{c}\text { kleine } \\
\text { Regierungs- } \\
\text { partei } \\
\text { (ohne SPD) }\end{array}$ & $\begin{array}{l}\text { große } \\
\text { Oppositions- } \\
\text { partei* }\end{array}$ & $\begin{array}{c}\text { Saldo Groß- } \\
\text { parteien } \\
\text { (in Klammern: } \\
\text { ohne Große } \\
\text { Koalitionen) }\end{array}$ \\
\hline 1953 bis 1980 & 1,93 & 1,91 & 0,10 & $-0,45$ & 1,21 & $3,40(3,61)$ \\
\hline 1983 bis 2009 & $-5,09$ & $-3,31$ & $-1,78$ & $-0,43$ & 1,01 & $-3,83(-2,57)$ \\
\hline 1953 bis 1965 & 2,13 & 4,15 & $-2,50$ & $-2,50$ & 2,53 & $6,68(6,68)$ \\
\hline 1969 bis 1980 & 1,73 & $-0,33$ & 2,05 & 1,60 & $-0,53$ & $0,13(-0,47)$ \\
\hline 1983 bis 1994 & $-3,93$ & $-3,00$ & $-0,93$ & $-0,93$ & 0,63 & $-2,38(-2,38)$ \\
\hline 1998 bis 2009 & $-6,25$ & $-3,63$ & $-2,63$ & 0,23 & 1,53 & $-5,28(-2,13)$ \\
\hline \multicolumn{7}{|c|}{$\begin{array}{l}\text { * Als große Oppositionsparteien wurden nur CDU/CSU und SPD gewertet, so dass Zeiten Großer } \\
\text { Koalitionen in dieser Spalte nicht einbezogen wurden. } \\
\text { Vgl. die Anmerkungen zu Abbildung } 1 \text { und } 2 . \\
\text { Quelle: Eigene Berechnung. }\end{array}$} \\
\hline
\end{tabular}

Die bisherigen Ergebnisse verdeutlicht auch noch einmal Tabelle 1, in der die durchschnittlichen Stimmenveränderungen für einzelne Zeitabschnitte ausgewiesen sind - einerseits für die beiden Hälften des Untersuchungszeitraums (acht Wahlen), andererseits für jeweils vier Wahlen. Sowohl für die Regierungskoalition insgesamt als auch die große Regierungspartei ist dabei für beide Zeitabschnittseinteilungen eine fortlaufende Verschlechterung der Durchschnittswerte erkennbar (Spalte 2 und 3).

Bezüglich der kleinen Regierungspartei zeigt sich dies hingegen nur, wenn man die ersten acht Folgewahlen mit den letzten acht vergleicht und dabei auch Große Koalitionen berücksichtigt (Spalte 4). Aber auch hier weist die gegenüberstellende Betrachtung von jeweils vier Wahlen schon eine gewisse Schwankung auf, mit positiven Durchschnittswerten für den zweiten Zeitraum (1969 bis 1980). Ohne die SPD als kleine Regierungspartei, das heißt ohne Große Koalitionen (Spalte 5), gibt es nahezu keine Unterschiede bei einer Zweiteilung des Untersuchungszeitraums. Betrachtet man die Zeitabschnitte für jeweils vier Wahlen, wird noch deutlicher, dass für den kleinen Regierungspartner kein Abwärtstrend existiert: Negative und positive Durchschnittswerte wechseln sich ab.

Überdies ist hinsichtlich der Großparteien mit Oppositionsstatus in Spalte 6, die Zeiten Großer Koalitionen folglich nicht einbezieht, keine durchgehende Abwärtsbewegung erkennbar. Es treten bis auf einen Zeitraum von vier aufeinanderfolgenden Wahlen (1969 bis 1980) auch immer durchschnittliche Stimmengewinne auf.

Der Saldo der Großparteien Union und SPD (Spalte 7) zeigt hingegen einen negativen Trend. Bei dessen Interpretation ist - wie allgemein auch bei den kumulativen Stimmenanteilen der Volksparteien im Längsschnitt - jedoch einerseits zu beachten, dass Verluste für die Kanzlerpartei bisweilen größer ausfielen als Gewinne der großen Oppositionspartei. 1983 und 1998 führte dies letztlich zu einem negativen Einzelergebnis. Andererseits hat im Zeitverlauf auch die Anzahl der Verlustwahlen für die Kanzlerpartei zugenommen, die ab 1983 immer verlor. In die Gesamtentwicklung fließt aber ein, dass oppositionelle Volksparteien seit 1987 eben nicht mehr so deutlich von Verlusten der regierenden Volkspartei pro- 
fitieren. ${ }^{15}$ Bezieht man hier im Übrigen Große Koalitionen, in denen beide Volksparteien zur Folgewahl als Regierungsparteien antraten, nicht mit ein (Spalte 7, in Klammern), zeigt sich die konstatierte Abwärtsbewegung nahezu unverändert.

\section{Erklärungsfaktoren}

Aus einer Vielzahl möglicher Einflussgrößen werden für den aufgezeigten Trend drei aufgeführt. Auch wenn diese teilweise die Volksparteien allgemein betreffen - der Fokus liegt im Folgenden vor allem auf den Rahmenbedingungen für Regierungsparteien.

\subsection{Der Aufstieg des kritischen Bürgers}

Es kann angenommen werden, dass ein Verlust an Zustimmung für Regierungsparteien im Zusammenhang steht mit einem generellen Rückgang an Vertrauen in politische Akteure und Institutionen. ${ }^{16}$ Zur Illustration der hier denkbaren Einflusseffekte lässt sich auf die von David Easton eingeführten Formen politischer Unterstützung (specific und diffuse support) verweisen: Die Kategorie der spezifischen Unterstützung stellt dabei auf die kurzfristig potentiell schwankende Zufriedenheit mit dem Output beziehungsweise den Leistungen der politischen Amtsträger (authorities) ab. Sie speist sich aus dem Vergleich der eigenen Ansprüche und Präferenzen mit den konkreten Handlungen und Ergebnissen der in Entscheidungsverantwortung stehenden Regierungsparteien. Demgegenüber bezieht sich der Begriff der diffusen Unterstützung bei Easton auf eine generalisierte Bewertung verschiedener Komponenten des politischen Systems (politische Amtsträger, Verfassungsordnung, politische Gemeinschaft), die weitgehend unabhängig von den (spezifischen) Outputs erfolgt und insofern stabiler ist. ${ }^{17}$ Langfristig kann die wahrgenommene Regierungsleistung allerdings den Auf- und Abbau von Vertrauen, das Easton später als eine Form von diffuser Unterstützung spezifizierte ${ }^{18}$, beeinflussen. Kenneth Newton und Pippa Norris konstatieren in dieser Hinsicht etwa „substantial support for theories that focus on the performance of governments to explain citizens declining confidence "19. Umgekehrt bewegt sich die verminderte Zustimmung für Regierungsparteien aber auch im Fahrwasser eines allgemeinen Vertrauensverlusts, der möglicherweise die Bewertung spezifischer Leis-

15 Zudem gilt für die Konstellation, dass auf eine Verlustwahl vier Jahre später eine Wahl mit Stimmengewinnen folgte: Während die späteren Gewinne bei der Kanzlerpartei immer kleiner als die vorherigen Verluste waren, war von diesem Muster bei der letzten einschlägigen Wahl - anders als in den Jahrzehnten zuvor - auch die oppositionelle Großpartei betroffen (siehe oben).

16 Vgl. zum Beispiel Russell J. Dalton, Democratic Challenges, Democratic Choices. The Erosion of Political Support in Advanced Industrial Democracies, Oxford 2004, S. 29 ff.

17 Vgl. David Easton, A Systems Analysis of Political Life, New York 1965, S. 268 ff.

18 Vgl. ders., A Re-Assessment of the Concept of Political Support, in: British Journal of Political Science, 5. Jg. (1975), H. 4, S. 435 - 457, S. 446 ff.

19 Kenneth Newton / Pippa Norris, Confidence in Public Institutions: Faith, Culture, or Performance?, in: Susan J. Pharr / Robert D. Putnam (Hrsg.), Disaffected Democracies, Princeton 2000, S. $52-73$, S. 72. 
tungen beeinflusst. ${ }^{20}$ Davon wären Regierungsparteien insofern besonders betroffen, als sie eine exponierte Position im politischen System einnehmen, vermutlich als seine ersten Repräsentanten wahrgenommen werden.

Schon seit einiger Zeit ist in der empirischen Demokratieforschung auch vom „Aufstieg des kritischen Bürgers" die Rede. Dieser zeichnet sich zwar durch eine hohe Bewertung der Demokratie als Ordnungsidee aus, zugleich aber durch wachsende Unzufriedenheit mit der konkreten Leistung eines politischen Systems. ${ }^{21}$ Ronald Inglehart bringt diese Entwicklung mit der Ausbreitung postmaterialistischer Werte in Verbindung, die eine kritischere Bewertung von Politik zur Folge haben. ${ }^{22}$ So gesehen handelt es sich also um „dissatisfied democrats" 23 , deren Unzufriedenheit sich auch bei Wahlen niederschlägt, in erster Linie bei den für die Systemleistung maßgeblichen Regierungsparteien.

Eine im Ergebnis ähnliche, aber in der Begründung andere Perspektive betont seit den 1970 er Jahren ansteigende Kosten-Nutzen-Kalküle beim Bürger, die vor allem als Spillover-Effekte eines hegemonialen neoliberalen Wirtschaftsmodells gedeutet werden. Demnach hat nicht nur eine Ausdifferenzierung der Präferenzen stattgefunden; ein Übergreifen ökonomisch rationalen Denkens auf die Sphäre der Politik hat vielmehr auch „den individuell wahrgenommenen Geltungsanspruch subjektiver Präferenzen radikal erhöht und damit die Frustrationstoleranz massiv reduziert "24. Gemäß dieser Lesart wird das politische System also viel stärker als Dienstleister wahrgenommen, der die eigenen Präferenzen zu berücksichtigen hat. Geschieht dies nicht, „verringert [sich] insbesondere die perzipierte Kompetenz der Institution ,Regierung “' 25 . Auch hiervon wären also in erster Linie Regierungsparteien betroffen.

In den Kategorien von Easton formuliert müssten sich im Angesicht kritischerer Bürger also die Bedingungen gewandelt haben, um spezifische Unterstützung zu generieren. Umgekehrt dürfte sich auf längere Sicht das Reservoir diffuser Unterstützung in dem Maße verringern, in dem diese von langfristigen Erfahrungen mit spezifischen Systemleistungen abhängt. ${ }^{26}$

Ein Blick auf die Bevölkerungseinstellungen in Deutschland zeigt eine im Zeitverlauf vergleichsweise konstante Zustimmung zur Demokratie als Ordnungsmodell (Werteebene) sowie zur durch das Grundgesetz ausgeformten Institutionenordnung (Strukturebene). Bei

20 Vgl. Suzanne S. Schüttemeyer, Bundestag und Bürger im Spiegel der Demoskopie, Opladen 1986, S. 50.

21 Vgl. Pippa Norris, Conlusions: The Growth of Critical Citizens and its Consequences, in: dies. (Hrsg.), Critical Citizens. Global Support for Democratic Governance, Oxford 1999, S. 257 272, S. 269.

22 Vgl. Ronald Inglehart, Postmodernization Erodes Respect for Authority, but Increases Support for Democracy, in: Pippa Norris (Hrsg.), a.a.O. (Fn. 21), S. 236 - 256, S. 250.

23 Hans-Dieter Klingemann, Mapping Political Support in the 1990s: A Global Analysis, in: Pippa Norris (Hrsg.), a.a.O. (Fn. 21), S. 31 - 56, S. 34, S. 54.

24 Gary S. Schaal, Responsivität - Selbstzerstörerisches Ideal liberaler Demokratie?, in: ders. / André Brodocz / Marcus Llanque (Hrsg.), Bedrohungen der Demokratie, Wiesbaden 2009, S. 353 369, S. 363 f., S. 366.

25 Ebenda, S. 354. Eine solche Annahme läuft dann auch nicht unbedingt der PostmaterialismusThese Ingleharts zuwider. Schaal betont vor allem eine steigende Intensität, mit der die Berücksichtigung eigener (wie auch immer gearteter) Präferenzen erwartet wird.

26 Allerdings ist zu beachten, dass diffuse Unterstützung sich bei Easton eben nicht nur auf Amtsträger, sondern alle drei oben genannten Objekte bezieht. 
der Bewertung der für den kritischen Bürger besonders relevanten Systemleistung (Performanzebene) gingen die Zufriedenheitswerte in den 1990er Jahren hingegen sichtbar zurück. Seitdem sind auf einem insgesamt niedrigeren Niveau größere zyklische Schwankungen zu verzeichnen. Zu bedenken ist allerdings, dass die deutsche Wiedervereinigung und ihre Folgen eine erhebliche Belastungsprobe für das politische System darstellten, außerdem eine (auch auf der Performanzebene) kritischere beziehungsweise weniger zufriedene Bevölkerung Ostdeutschlands hinzukam. ${ }^{27}$ Ins Bild des „kritischen Bürgers“ passen zudem empirische Daten, wonach die „external efficacy“ - der Glaube der Bürger, dass Politiker auf ihre Anliegen reagieren - seit den 1970er Jahren sinkt, während die „internal efficacy“ - der Glaube, Einfluss ausüben zu können - gestiegen ist. ${ }^{28}$

Dass die tatsächliche Leistung eines politischen Systems, gemessen an empirischen Indikatoren, entgegen mancher Krisensemantik im Zeitverlauf nicht gesunken ist ${ }^{29}$, spielt im hier behandelten Zusammenhang im Übrigen keine gewichtige Rolle. Auch ein teilweise feststellbarer Anstieg an Effektivität kann im Urteil kritischerer Bürger zu einem Rückgang an wahrgenommener Leistungsfähigkeit und geringerer Zufriedenheit mit Regierungsparteien führen.

\subsection{Wandel im Wählerverhalten und im Parteiensystem}

Auch wählersoziologische Veränderungen haben zu veränderten politischen Bedingungen geführt. So wurden insbesondere parteispezifische Milieubindungen durch Prozesse wie Säkularisierung, sektoralen Wandel und Wertewandel in hohem Maße „wegmodernisiert" ${ }^{\text {“30. }}$ Sowohl die Größe der Milieus als auch ihre Prägekraft für das Wahlverhalten haben sich in der Folge erheblich verringert. ${ }^{31}$

Schlägt sich dies letztlich in einem größeren Wechselwähleranteil und höherer Volatilität nieder $^{32}$, liegt die unmittelbare Folge für die Parteien darin, die Bürger immer wieder neu

27 Vgl. insgesamt Oskar Niedermayer, Bevölkerungseinstellungen zur Demokratie: Kein Grundkonsens zwischen Ost- und Westdeutschen, in: ZParl, 40. Jg. (2009), H. 2, S. 383 - 397, S. 387, S. $392 \mathrm{f}$.

28 Vgl. Angelika Vetter, Frischer Wind in einer alten Beziehung? Political Efficacy und die Bundestagswahl 1998, in: Jürgen Falter / Oscar W. Gabriel / Hans Rattinger (Hrsg.), Wirklich ein Volk?, Opladen 2000, S. 79 - 109, S. 80.

29 Edeltrand Roller zeigt dies in einer Untersuchung von 21 OECD-Staaten explizit auch für Deutschland; dies., The Performance of Democracies. Political Institutions and Public Policies, Oxford 2005, vgl. für Deutschland etwa S. 181, S. 215, S. 273.

30 Peter Lösche, a.a.O. (Fn. 9), S. 9. Vgl. allgemein Seymour Martin Lipset / Stein Rokkan, Cleavage Structures, Party Systems and Voter Alignments. An Introduction, in: dies. (Hrsg.), Party Systems and Voter Alignments. Cross-National Perspectives, New York 1967, S. $1-64$.

31 Dies zeigt sich etwa daran, dass die Arbeiterschaft, als das klassische Unterstützermilieu der SPD, bei der Bundestagswahl 2009 „fast gleichermaßen“ für linke wie für rechte Parteien votierte. Richard Hilmer, a.a.O. (Fn. 9), S. 168.

32 So verzeichnete die Bundestagswahl 2009 zum Beispiel Rekordmarken bei der Netto-Volatilität (diese war nur 1953 größer) und beim Wechselwähleranteil. Vgl. ebenda, S. 149. Beide Größen sind auch auseinanderzuhalten. Der Wechselwähleranteil gibt an, wie viele Wähler bei aufeinanderfolgenden Bundestagswahlen ihre Stimme unterschiedlichen Parteien gegeben haben, wird also über demoskopische Befragungen ermittelt. Die Volatilität beschreibt hingegen die Wählerwanderung im Aggregat, das heißt die Netto-Veränderung für die Parteien zwischen zwei Wahlen (auch 
überzeugen zu müssen. Dies gilt nun allerdings für Regierungs- und Oppositionsparteien gleichermaßen. Gerade Union und SPD sind aber stärker davon betroffen, wenn die Parteibindung als Wahlmotiv gegenüber Kandidaten- und Themenorientierung an Bedeutung verliert. Zumindest spielt die Parteiidentifikation für die Kleinparteien eine deutlich geringere Rolle; für ihre Wähler sind primär inhaltliche Fragen wichtig. ${ }^{33}$ Insofern ist - einerseits - ein Bindungsrückgang besonders für die Großparteien problematisch. Zudem stehen sie weiterhin vor der Herausforderung, als mitgliederstarke Parteien eine größere Heterogenität an Positionen zu mehrheitsfähigen Konzepten bündeln, also eine größere interne Integrationsleistung erbringen zu müssen. Andererseits ist es unter veränderten Rahmenbedingungen auf der Angebotsseite der Politik (siehe 2.3.) insgesamt gerade für regierende Parteien schwieriger, jene Überzeugungsarbeit zu leisten. In der Summe würde dies dann also für die vom Abschmelzen der Milieus besonders betroffenen und zugleich regierenden Großparteien umso mehr gelten.

Wenn sich angesichts einer Gesellschaftsformation, die sich durch Individualisierungstendenzen auszeichnet, Interessen und Präferenzen weniger an soziokulturellen Kontexten und vormals stabilen Gruppenzugehörigkeiten aller Art festmachen lassen, ist dies indessen nicht nur im Moment der Wahl von besonderer Bedeutung, sondern auch für Regierungshandeln während der Wahlperiode. Pluralisierung und Ausdifferenzierung der Präferenzen und deren sich daraus ergebende Unübersichtlichkeit verschlechtern für die Zeit des Regierens strukturell auch die Möglichkeit responsiven Handelns. ${ }^{34}$ Dies gilt letztlich zwar wieder für alle, insbesondere aber für in Entscheidungsverantwortung stehende Regierungsparteien. Zu der vom Repräsentationsprinzip geforderten Notwendigkeit einer Eigenleistung auf Seiten der Repräsentanten gehört in diesem Sinne eben auch, das zu repräsentierende Interesse überhaupt erst zu identifizieren.

Überdies kommen als Erklärungsfaktor auch Veränderungen im Parteiensystem insgesamt in Betracht. Insbesondere der dauerhafte Einzug der Grünen in den Bundestag seit der Wahl von 1983 stellt hier einen Einschnitt dar. Einerseits war für den Wähler damit eine Pluralisierung im Angebot der parlamentarisch etablierten Parteien verbunden. Andererseits kam mit der zusätzlichen Bundestagsfraktion nun auch die konsensuelle parlamentarische Praxis der 1960er und 1970er Jahre zu einem Ende. ${ }^{35}$ In der Folge sank nicht nur der Anteil einstimmig verabschiedeter Gesetze von circa 70 auf 15 Prozent. Die zweite Oppositionsfraktion belebte auch den inneroppositionellen und damit insgesamt den parlamentarischen Parteienwettbewerb und führte am Ende in eine „deutlich kompetitivere Gestalt des Parlamentarismus“36. Eine ähnliche Veränderung der Angebots- und Wett-

Netto-Volatilität genannt). Sie dient als Indikator für die Dynamik im Parteiensystem und wird zumeist nach Mogens N. Pedersen berechnet als Summe der Veränderung der Anteile aller Parteien geteilt durch zwei. Vgl. ders., The Dynamics of European Party Systems: Changing Patterns of Electoral Volatility, in: European Journal of Political Research, 7. Jg. (1979), H. 1, S. 1 - 26.

33 Vgl. Richard Hilmer, a.a.O. (Fn. 9), S. 168.

34 Vgl. Gary S. Schaal, a.a.O. (Fn. 24), S. 361.

35 Vgl. Suzanne S. Schüttemeyer, 50 Jahre deutscher Parlamentarismus: Kategorien und Kriterien für Leistungen und Defizite, in: Thomas Ellwein / Everhard Holtmann (Hrsg.), 50 Jahre Bundesrepublik Deutschland. Rahmenbedingungen - Entwicklungen - Perspektiven, Opladen 1999, S. 482 -495 , S. 490 f.

36 Ebenda, S. 491. 
bewerbsstruktur ergab sich später insbesondere mit der Etablierung der PDS beziehungsweise der Linkspartei als gesamtdeutsche Partei. ${ }^{37}$ In diesem Zusammenhang stand nach der Bundestagswahl 1998 eine Regierungskoalition (Rot-Grün) auch erstmals seit den 1950er Jahren wieder einer „zweiseitigen“ Opposition (der PDS auf der linken, FDP und Union auf der rechten Seite) gegenüber.

Dass die genannten Veränderungen im Parteiensystem durchaus als Erklärungsgröße für den oben aufgezeigten Trend in Frage kommen, zeigt sich im Übrigen auch an der in den empirischen Daten deutlich gewordenen Zweiteilung des Untersuchungszeitraums in eine Periode bis 1980 und ab 1983. In dieser Hinsicht stellte die Wahl von 1983 cum grano salis also nicht nur im programmatischen Anspruch der neuen christdemokratisch-liberalen Koalition eine „Wende“ dar.

\subsection{Postnationale Konstellation, Komplexitätswachstum und die Politik der Zumutungen}

Während neben der Pluralisierung im Parteiensystem bisher vor allem nachfrageseitige Veränderungen und ihre Folgen angesprochen wurden, kann ein Wandel der Bedingungen für Regieren auch mit Blick auf die Angebotsseite von Politik festgestellt werden. ${ }^{38}$

(1) Verwiesen werden kann zunächst auf die nunmehr „postnationale Konstellation“, in der (national-)staatliche Handlungsfähigkeiten eingeschränkt und die Legitimationsketten politischen Handelns brüchig werden, zumindest sich aber über den nationalstaatlichen Raum hinaus verlängern. ${ }^{39}$ Ein prominentes Beispiel hierfür wäre etwa Politik im Rahmen der Europäischen Union, die unter der Beteiligung einer Vielzahl von Akteuren mit den entsprechenden Legitimations- und Transaktionskosten ausgehandelt wird.

Dahinter steht einerseits die Vermutung, dass Regierungen beziehungsweise Regierungsmehrheiten in geringerem Maße Entscheidungen autonom treffen können, für diese aber weiterhin im gleichen Maße verantwortlich gemacht werden. Zum Trend der vielschichtigen „Zerfaserung" von Staatlichkeit gehört in diesem Sinne auch eine „Entbündelung" von Verantwortung: Während die Entscheidungsverantwortung seit den 1970er Jahren mehr und mehr internationalisiert, Organisationsverantwortung mehr und mehr privatisiert wurde, verbleibt die Letztverantwortung doch beim Staat. ${ }^{40}$ De facto bedeutet Staat dabei aber

37 Zumindest mit Blick auf die Bundestagswahl 2009 kann davon gesprochen werden, dass die Linkspartei den Schritt zu einer gesamtdeutschen Partei vollzogen hat. So blieb sie 2009 in nur sieben westdeutschen Wahlkreisen unter fünf Prozent. Vgl. Richard Hilmer, a.a.O. (Fn. 9), S. 164.

38 Als mögliche Erklärungsgröße kommt in dieser Hinsicht theoretisch auch ein kontinuierlicher Qualitätsverlust der regierenden Elite in Betracht. Unabhängig davon, wie dieser überhaupt empirisch messbar wäre, wird hier bezüglich der Qualitätsmerkmale des Regierungspersonals eine gewisse Konstanz unterstellt. Es wird also zum Beispiel nicht davon ausgegangen, dass es sich heute um eine systematisch weniger fähige politische Elite handelt.

39 Vgl. etwa Jürgen Habermas, Die postnationale Konstellation und die Zukunft der Demokratie, in: ders. (Hrsg.), Die postnationale Konstellation, Frankfurt am Main 1998, S. 91 - 169.

40 Vgl. Philipp Genschel / Stephan Leibfried / Bernhard Zangl, Der zerfasernde Staat. Vom Wandel des Subjekts moderner Politik, in: Dieter Wolf (Hrsg.), Staat und Gesellschaft - fähig zur Reform?, Baden-Baden 2007, S. 37 - 45, S. 40 ff. 
die jeweiligen Regierungen. Gerade diese sind betroffen, wenn der Staat als „universaler Ausfallbürge“ in der Öffentlichkeit „in Haftung genommen wird“, auch weil „diese Verantwortungszuschreibung den tatsächlichen Handlungsmöglichkeiten nicht mehr gerecht [wird] “ ${ }^{41}$. Andererseits lässt sich in diesem Zusammenhang annehmen, dass mit einem möglichen Verlust an (national-)staatlicher Handlungsfähigkeit eben kein Rückgang an Anspruchshaltungen und Leistungserwartungen an den Staat einhergeht. Gerade vor dem Hintergrund kritischerer Bürger würde eine solche Entwicklung Regieren „schwieriger“ machen. ${ }^{42}$

(2) Einen anderen Aspekt verdeutlicht die Rede von einem Komplexitätswachstum im Bereich des Politischen. Dieses ergibt sich demnach einerseits aus einer Verflechtung der Problemzusammenhänge im Gefolge fortschreitender Globalisierung. Als Herausforderungen par excellence können die aktuelle europäische Finanzkrise und speziell die Unterstützung Griechenlands gelten, bei der politischen Lösungsvorschlägen angesichts der Unsicherheit über ihre Wirksamkeit und Folgen durchaus experimenteller Charakter zukommt. ${ }^{43}$ Zur Komplexität und Tragweite hinzu kommt die Durchsetzbarkeit entsprechender Problemlösungen nicht nur im eigenen Land, sondern auch auf europäischer Ebene. Andererseits steht dahinter, auch wenn man nur die nationale Ebene betrachtet, eine allgemeine Entwicklungstendenz moderner ausdifferenzierter Gesellschaften, die sich durch eine Vielzahl politisch relevanter und häufig interdependenter Problemfelder auszeichnen: „The growing complexity of the world of politics [...] results not only from increasing and global interdependencies, but from the very expansion of the sphere of politics." ${ }^{44}$ Eine „burden of complexity“ lastet demzufolge aber nicht nur auf Regierungen, die über immer komplexere Sachverhalte entscheiden müssen; ebenso betroffen sind die Bürger im Hinblick auf ihre Fähigkeit, Regierungshandeln und die anstehenden politischen Fragen selbst zu beurteilen. ${ }^{45}$

Aus einer anderen Perspektive lässt sich in dieser Hinsicht auch die veränderte Zeitdimension moderner Politik betonen. Danach wächst mit dem Umfang der entscheidungsbedürftigen Materien und deren (Re-)Regulierungstempo eben nicht nur der Bedarf an Zeit für (demokratische) Willensbildung und Entscheidungsfindung. Es ist gerade ein gegenläufiges Kennzeichen der modernen „beschleunigten Demokratie“, dass die für Entscheidungen zur Verfügung stehende Zeit tendenziell abnimmt. ${ }^{46}$ Auch hierfür bietet

41 Ebenda, S. 42, S. 44

42 Inwiefern tatsächlich ein Verlust an staatlicher Handlungsfähigkeit festgestellt werden kann, ist allerdings eine empirisch offene Frage. Gemessen anhand verschiedener Effektivitätsindikatoren kann Edeltraud Roller auch für die jüngere Periode der 1990er Jahre keinen generellen Rückgang an staatlicher Leistungstätigkeit nachweisen. Dies., a.a.O. (Fn. 29), S. 193, S. 237.

43 Vgl. auch Wolfgang Streeck, The Crisis of Democratic Capitalism, in: New Left Review, 52. Jg. (2011), H. 5, S. 5 - 29, S. 25 f.

44 Giovanni Sartori, The Theory of Democracy Revisited, Chatham 1987, S. 120.

45 Vgl. Russell Hardin, The Public Trust, in: Susan J. Pharr / Robert D. Putnam (Hrsg.), a.a.O. (Fn. 19), S. $31-51$, S. 42 , S. 47 f.

46 Vgl. Hartmut Rosa / Henning Laux, Die beschleunigte Demokratie - Überlegungen zur Weltwirtschaftskrise, in: WSI-Mitteilungen, 62. Jg. (2009), H. 10, S. 547 - 553. Weil Globalisierung in dieser Hinsicht vor allem „Beschleunigung“ bedeutet, steht am Ende die ernsthafte Gefahr einer De-Synchronisierung zwischen Politik und anderen sozialen Systemen (wie Wirtschaft und Medien): Demokratie ist damit „in danger of falling victim to the powers of speed. Its temporal patterns appear to be irreconcilably out of step with the time structures of the global age". 
die Finanzkrise im Euro-Raum ein gutes Beispiel. Demokratische Politik bleibt eben auch in einem dynamischeren Umfeld auf zeitlich aufwändige Steuerungsprozesse angewiesen. Unter den Bedingungen von Komplexität und Zeitdruck können die Erwartungen an die Politik dann eher enttäuscht werden, weil Entscheidungen aus der Output-Perspektive nicht schnell genug oder aus der Input-Perspektive zu schnell getroffen werden. ${ }^{47}$ Überdies verringert sich tendenziell auch die Zeitspanne, um politische Entscheidungen nachhaltig zu vermitteln.

(3) Es kann vermutet werden, dass Regieren gerade unter dem Vorzeichen einer die öffentliche Debatte dominierenden "Politik der Zumutungen“" schwieriger geworden ist. War in früheren Jahrzehnten immer auch ein Ausbau des sozialstaatlichen Wohlfahrtsniveaus prägend für den Parteienwettbewerb, haben geänderte Rahmenbedingungen die Parteien nunmehr vor die Herausforderung gestellt, auch ohne eine (angekündigte oder umgesetzte) Politik der Wohltaten reüssieren zu müssen. ${ }^{48}$ Damit ist es nicht nur schwerer, kollektiv verbindliche Entscheidungen zu treffen, sondern gerade auch Akzeptanz für sie zu schaffen.

Dahinter steht wiederum ein schrumpfender finanzpolitischer Handlungsspielraum, der sich neben anderen Faktoren aus einer wachsenden Staatsverschuldung und - in Reaktion darauf - dem „schmerzhafte(n) Übergang in ein Regime fiskalischer Austerität“49 ergibt. Die Konsolidierung der öffentlichen Haushalte ist mittlerweile auch in Deutschland zu einem wichtigen Thema der politischen Auseinandersetzung geworden - und mit der so genannten Schuldenbremse nunmehr auch ein mit Verfassungsrang ausgestattetes Gebot. Allerdings dürfte es der christdemokratischen Union nicht so viel leichter fallen als der sozialdemokratischen Konkurrenz, den Weg der Konsolidierung und zu einem „schlanken“ Staat zu gehen, denn auch sie kann als Sozialstaatspartei gelten. ${ }^{50}$

Die „Politik der Zumutungen“ dürfte ausweislich der oben aufgezeigten empirischen Daten für die den Kanzler stellenden Volksparteien als „,geborene“ Regierungsparteien“51 besonders prekär sein, denn sie werden tatsächlich stärker für eine Regierungsleistung in Haftung genommen. Hiermit übereinstimmend errangen vor allem die Kanzlerparteien anfangs noch beachtliche Stimmenzuwächse, gerade weil ihnen die - seinerzeit positiv ausfallende - Regierungsleistung stärker zugeschrieben wurde. Später, bei ungünstigeren Verteilungsbedingungen, fiel es ihnen hingegen zunehmend schwerer, während die jeweiligen Juniorpartner durchaus noch Gewinne verzeichnen konnten - vermutlich auch, weil kleine

Hartmut Rosa, The Speed of Global Flows and the Pace of Democratic Politics, in: New Political Science, 27. Jg. (2005), H. 4, S. 445 - 459, S. 454.

47 Zugleich ist handelnde Politik dabei auch immer wieder „zur aktiven Reproduktion der Rationalitätsfunktion gezwungen“, sie muss zumindest den Anschein erwecken, genau um die (beabsichtigten) Konsequenzen ihres Handelns zu wissen. Hartmut Rosa / Henning Laux, a.a.O. (Fn. 46), S. 551.

48 Vgl. Elmar Wiesendahl, Parteien und die Politik der Zumutungen, in: APuZ, B 40 / 2004, S. 19 -24 .

49 Wolfang Streeck, Noch so ein Sieg, und wir sind verloren. Der Nationalstaat nach der Finanzkrise, in: Leviathan, 38. Jg. (2010), H. 2, S. 159 - 173, S. 163.

50 Vgl. Manfred G. Schmidt, Wenn zwei Sozialstaatsparteien konkurrieren: Sozialpolitik in Deutschland, in: ders. / Reimut Zohlnhöfer (Hrsg.), Regieren in der Bundesrepublik Deutschland: Innenund Außenpolitik seit 1949, Wiesbaden 2006, S. 137 - 157.

51 Everhard Holtmann, a.a.O, (Fn. 8), S. 222. 
Parteien weniger für Regierungsleistungen beziehungsweise einschneidende Sozialreformen verantwortlich gemacht werden. ${ }^{52}$

Das elektorale Dilemma der regierenden Volksparteien besteht insofern darin, sich entweder einem Reformdruck zu verweigern und die Verantwortung für Folgeprobleme zu übernehmen, oder aber die eigene Wiederwahl durch eine unpopuläre Politik sozialer Einschnitte zu gefährden. ${ }^{53}$ Hinzu kommt, dass das deutsche Parteiensystem mittlerweile eine Akteursstruktur ausgebildet aus, die auf der den Wettbewerb so prägenden sozialstaatlichen Konfliktlinie nunmehr zwei gesamtdeutsch etablierte Parteien ausweist, die die Pole Marktfreiheit einerseits, soziale Gerechtigkeit andererseits - repräsentieren. Nicht mehr nur besitzen von der Union enttäuschte wirtschaftsliberale Wähler mit der FDP eine weitere elektorale Handlungsoption; seit der deutschlandweiten Etablierung der Linkspartei gilt dies auch für mit der SPD unzufriedene sozialstaatsaffine Wähler. ${ }^{54}$ Weil es sich bei allen genannten Aspekten um angebotsseitige Erklärungsfaktoren handelt, sind auch hiervon wieder in erster Linie Regierungsparteien betroffen. ${ }^{55}$

\section{Quo vadetis (regierende) Volksparteien?}

Der festgestellte Trend zunehmender Stimmenverluste besonders für die Kanzlerpartei ist gewiss kein ehernes Gesetz - dass er sich aber in absehbarer Zeit umkehrt, ist eher fraglich. Zwar vollzieht sich jene Entwicklung in einem generell für Volksparteien ungünstigen Umfeld. Aber auch vor dem Hintergrund, dass Regierungen in so genannten Nebenwahlen zunehmend abgestraft werden ${ }^{56}$, scheint das Geschäft des Regierens zumindest „schwieriger" geworden zu sein.

Zwei Aspekte sind bei der Bewertung obiger Befunde indes zu berücksichtigen. Einerseits können für diese gerade strukturelle Gründe verantwortlich gemacht werden. Beliebt, aber allzu einfach ist demnach die Deutung, jene Entwicklung sei primär durch die Parteien selbst verursacht und müsse ihnen als „Versagen“ zum Vorwurf gemacht werden. ${ }^{57}$ Andererseits ist die plakative Aussage, dass Regieren „schwieriger“ geworden sei, normativ kei-

52 Unabhängig davon wurde gerade der FDP als kleiner Koalitionspartei bei der Regierungsleistung aber viele Jahrzehnte eine Korrektivfunktion zugeschrieben.

53 Vgl. Armin Schäfer, Die Reform des Sozialstaates und das deutsche Parteiensystem: Abschied von den Volksparteien?, in: ZParl, 38. Jg. (2007), H. 3, S. 648 - 666, S. 649; Elmar Wiesendahl, a.a.O. (Fn. 48), S. 20.

54 Vgl. Oskar Niedermayer, Von der Zweiparteiendominanz zum Pluralismus: Die Entwicklung des deutschen Parteiensystems im westeuropäischen Vergleich, in: PVS, 51. Jg. (2010), H. 1, S. 1 13, S. 11.

55 In der vorliegenden Untersuchung ging es nur um die relative Zustimmung derer, die tatsächlich zur Wahl gehen. Nicht auszuschließen sind Effekte, die sich aus anwachsenden Nichtwählerzahlen ergeben, vor allem wenn diese aufgrund von „Enttäuschungserfahrungen“ (Richard Hilmer, a.a.O. (Fn. 9), S. 176) zu Lasten der jeweiligen Regierungsparteien gehen.

56 Vgl. Michael Marsh, a.a.O. (Fn. 5), S. 606.

57 Ähnlich auch Peter Lösche für die genannten wählersoziologischen Änderungen. Vgl. ders., a.a.O. (Fn. 9), S. 9. Interessant ist in diesem Zusammenhang zudem, ob sich ähnliche Tendenzen auch in anderen westeuropäischen Staaten feststellen lassen. Eine entsprechende Entwicklung würde darauf hindeuten, dass es sich nicht allein um „hausgemachte“ Probleme von Regierungsparteien handelt. 
neswegs per se problematisch, denn Stimmenverluste können zumindest als eine demokratische Normalität begriffen werden. Differenziert zu betrachten ist allerdings, dass das Wechselverhältnis zwischen regierenden und opponierenden Volksparteien, zwischen Regierungsmalus und Oppositionsbonus teilweise brüchig geworden ist.

Über welche Möglichkeiten verfügen nun aber Großparteien, und gerade die besonders betroffenen regierenden Volksparteien, mit jenen (strukturellen) Entwicklungen umzugehen? Wichtig ist zunächst, dass Union und SPD trotz deutlicher Schrumpfung des Stammwähleranteils auch heute noch ein erhebliches Wählerpotential besitzen, „das weit über ihre

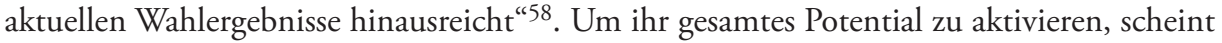
es dabei einerseits notwendig, die Integration parteispezifischer Milieus nicht zu vernachlässigen. Letztere mögen zwar abschmelzen, sind aber gleichwohl noch wählerwirksam vorhanden. Andererseits ist es für die Großparteien entscheidend, auf die mit ihnen jeweils verbundene Kernkompetenz, deretwegen sie in erster Linie gewählt werden, und eine hierzu komplementäre Sekundärkompetenz zugleich zu setzen. ${ }^{59}$ Dass beide Volksparteien, in der Marketing-Sprache formuliert, jeweils zwei so genannte Markenkerne (Wirtschaft und Soziales) besitzen, ist schon allein begrifflich problematisch. Faktisch kommt es aber darauf an, auch die jeweilige Sekundärkompetenz nicht zu vernachlässigen und sie letztlich glaubwürdig über prominente Köpfe zu personalisieren. Eine „erneuerte“ Parteibindung an die Großparteien wird in diesem Sinne mehr „kompetenzbasiert“ als „milieugestützt“ sein müssen. ${ }^{60}$

Darüber hinaus ist - unabhängig von der Frage, ob sich die Diskrepanz zwischen dem (autonomen) Handlungsspielraum nationaler Regierungen und den Anspruchshaltungen der Bürger vergrößert - auch von Bedeutung, wie sich die Rhetorik regierender Politiker zu den oben genannten Entwicklungen verhält. Zwar wird Politik im heutigen Medienzeitalter häufig mit dem Vorwurf unzureichender Kommunikation konfrontiert. An vielen Stellen mag die dahinterstehende Mahnung, die Erforderlichkeit politischer Maßnahmen umfassender zu begründen, auch zutreffen. Besonders wichtig dürfte in diesem Zusammenhang sein, dass - in Anlehnung an Ernst Fraenkel ${ }^{61}$ - die regierende politische Elite sich nicht in einer „Ideologie des Regierungsperfektionismus“ verliert. Enttäuschungs- und Frustrationserfahrungen entstehen in Zeiten wachsender Komplexität nämlich auch dadurch, dass zu hohe Erwartungen geweckt werden. Gerade Regierungen beziehungsweise Regierungsparteien laufen Gefahr einer „normativen Selbstüberforderung“62, wenn eine insofern ernüchternde Regierungspraxis jene Erwartungen nicht erfüllen kann. Ein weniger vollmundiger beziehungsweise strategisch sensibler Umgang mit Ankündigungen kann hier durchaus lohnend sein, auch wenn dies im öffentlichen Diskurs unter Konkurrenzbedingungen schwierig erscheint. ${ }^{63}$

58 Thomas Petersen, Das Potential der Volksparteien, in: FAZ vom 30. September 2009, S. 10.

59 Vgl. Oskar Niedermayer, a.a.O. (Fn. 54), S. 9.

60 Everhard Holtmann, a.a.O. (Fn. 8), S. 233.

61 Vgl. Ernst Fraenkel, a.a.O. (Fn. 3), S. 106.

62 André Brodocz / Marcus Llanque / Gary S. Schaal, Demokratie im Angesicht ihrer Bedrohungen, in: dies. (Hrsg.), a.a.O. (Fn. 24), S. $11-26$, S. 21.

63 Aber auch eine fortdauernde drastische Kritik der Opposition, die jeweilige Regierungspolitik sei „ein Turbolader für Politikverdrossenheit“ (Interview mit Sigmar Gabriel, in: Passauer Neue Presse vom 9. Juli 2011, S. 2), besitzt zumindest ein gewisses „Bumerang-Potential“. 
Zudem könnte es auch eine gewinnbringende Strategie sein, selbstbewusster und offensiver mit den besonderen politisch-sozialen Integrationsleistungen einer Volkspartei zu werben. Man kann in diesem Sinne mit guten (normativen) Gründen behaupten, dass sie die „fortschrittlichste Organisationsform politischer Willensbildung "64 darstellen. Auch wenn ein entsprechendes Handeln in einer komplexeren Gesellschaft nicht einfacher geworden ist - ausgestattet mit dem Anspruch und Selbstverständnis, sich für die gesamte Gesellschaft verantwortlich zu fühlen, liegt hier möglicherweise eine „Wachstumsperspektive“ für die Volksparteien. ${ }^{65}$

Wählt man den Bürger als Anknüpfungspunkt, lässt sich umgekehrt im Übrigen auch argumentieren, dass kritischere, kognitiv mobilisierte und im politischen Diskussionsprozess hierfür sensibilisierte Bürger einige der genannten strukturellen Wandlungsprozesse und deren Folgen auch selbst besser einordnen können beziehungsweise, dass man dies von kritische(re)n Bürgern erwarten kann.

Letztlich sind eben nicht nur politische Eliten damit konfrontiert, dass demokratische Politik nicht frei von Widersprüchen ist. Dies gilt mit Blick auf Idee und Praxis demokratischer Repräsentation zum Beispiel auch insofern, als demokratienotwendige Führung und ebenso demokratienotwendige Gefolgschaft nicht zugleich maximiert werden können. Relativ einfach lässt sich folglich die überlegene Position einnehmen, politischen Akteuren auch und gerade Regierungsparteien - immer genau den Teil vorzuwerfen, den sie gerade weniger erfüllen. Einseitig und dauerhaft aufzulösen ist das hier auftretende Spannungsverhältnis auf demokratischem Wege jedenfalls nicht ${ }^{66}$ - zumal sich wegen der oben beschriebenen Entwicklungen die Rahmenbedingungen sowohl für responsives Handeln als auch für politische Führungsleistungen eher verschlechtert haben.

64 Hans-Joachim Veen, Volksparteien: Die fortschrittlichste Organisationsform der politischen Willensbildung, in: ZParl, 30. Jg. (1999), H. 2, S. 377 - 381.

65 Vgl. Warnfried Dettling, 2010, Wachstumsperspektiven für eine Volkspartei, in: Forschungsjournal NSB, 23. Jg. (2010), H. 1, S. $64-66$.

66 Repräsentation ist in dieser Hinsicht konstitutiv „durch Mangel gekennzeichnet“. Zugleich indizieren Mangelerscheinungen in die eine oder andere Richtung aber auch nicht sofort eine „Krise“ der Repräsentation. Vielmehr können solche Strukturspannungen als „Lebenselixier pluralistischer Gesellschaften“ verstanden werden (so Uwe Thaysen, Repräsentative Demokratie: Ist der deutschen Bundestag dem zunehmenden Pluralismus noch gewachsen?, in: Günther Rüther (Hrsg.), Repräsentative oder plebiszitäre Demokratie - eine Alternative?, Baden-Baden 1996, S. 223 - 243, S. 227). Darüber hinaus fungieren sie in dieser dialektischen Perspektive aber letztlich auch als Lebenselixier des Repräsentationsprinzips selbst. Dies gilt gerade auch mit Blick auf eine in regelmäßigen Abständen (zuletzt wieder im Lichte der Proteste für und gegen das Bauprojekt „Stuttgart 21“) anschwellende Debatte über Instrumente direkter Demokratie. 